\title{
Application of Physical System Simulation Technology in volleyball Multimedia Courseware
}

\author{
Yipai Jiang ${ }^{1, a,{ }^{*},}$ Yanxiu Wang ${ }^{1}$ \\ ${ }^{1}$ College of Humanities and Social Sciences, Jingdezhen Ceramic Institute, Jingdezhen, Jiangxi, \\ China
}

ajyp333000@126.com

Keywords: Sports systems, simulation technology, volleyball, multimedia courseware.

\begin{abstract}
In this paper, the characteristics of Volleyball were demonstrated and analyzed, which shows professional, normative and contingency Volleyball Volleyball is tremendous room for growth, because volleyball with flexible teaching diversity, therefore multimedia technology into the volleyball classroom teaching, it fully mobilize the students 'interest in learning, to enhance the students' motivation to learn, more importantly, it really expands the students to learn volleyball divergent thinking, students learn to add endless charm.
\end{abstract}

\section{Introduction}

Volleyball flexible requires body movement coordination ability, therefore, since the campus since its promotion, by the majority of the students loved. However, because of the high standardization requirements volleyball and standardized operation, especially in the volleyball competition, volleyball players were asked to technology used in volleyball during a decisive run in the volleyball rules, effective action to complete the combination of offensive and defensive, and good coordination and cooperation between the players, therefore, the combination of technical teaching volleyball teaching the more requires the use of multimedia technology [1].

"Computer simulation of the audience not to panic, let the Olympic venues safer," the researchers Vienna sports training facilities equipment research center Pite Jia. Friedman said: "The timely detection of computer simulation to avoid a similar disaster Iser Mountain Stadium." It is for this purpose, simulation systems and technologies related to sports researchers are being pursued, and, in the various countries of public attention the efforts of sports researchers, sports simulation systems and technology has made many achievements, by researchers in relevant aspects of sports simulation systems and technology to sum up the achievements and do envisage on this basis, we hope to give some help volleyball teaching [2].

\section{Physical System Simulation Technology}

The rapid development of systems science for sports system simulation set up to lay a solid theoretical foundation. Physical system simulation technology is based on similar principles, information technology, systems technology and related professional and technical applications as the basis for computer equipment and a variety of physical effects as a tool to take advantage of the system model to actual or contemplated conduct experimental research system a comprehensive technology [1].

Applications sports system simulation technology has not only limited to test experimental sports activities, sports simulation technology has been expanded to be applicable to all areas of sports, including sports demonstration program, sports tactical and technical feasibility studies, design and analysis, testing, various stages of training [2]. Physical system simulation technology is not just for simple single system, also used in complex systems composed of a plurality of systems integration.

Significance of sports in volleyball teaching system simulation technology, Training. The 26th Congress of the International Volleyball Federation has adopted a new first rules of the game, its main contents are: per RALLY, 5 Board 3 wins system, with 25 points per game, no maximum limit 
points, get 25 points and lead the two into win a game. Deciding game of 15 points, no maximum limit points, get 15 points and lead into victory two council. The reform will give the volleyball rules bring some new influences and changes.

Physical system simulation technology as a new science and technology that the modern volleyball teaching and training to provide new ideas and teaching platform, it not only broke the limits of time and space so that we can at any angle or multi-angle viewing and demonstrate scientific, rational, standardized technical operation excellent volleyball players to be continuously and dynamically demonstrate tactical coordination, thus avoiding the traditional volleyball demonstrations in teaching and training deficiencies and overcome the static volleyball expressed in the form of textbooks dynamic volleyball skills, lack of content on tactics [3].

Volleyball simulation technology, three-dimensional animation tactical action. Virtual simulation animation is generally through motion capture system for Elite Volleyball Players collect and record, then motion track attached to the virtual character animation for simulation. But the device motion capture system and excellent sports are very expensive to borrow, which makes the cost of research and animated bear [4].

This is the technology in the race action sequences as a template to simulate animation production by trimming excellent volleyball players, these games technical action sequences as the camera angle, distance, shelter and other factors difficult to direct, complete and clear display of technical movements [2]. However, these technical movements as a template with a virtual imitation of the player characters to achieve volleyball virtual simulation animation technology, you can watch movies in three dimensions and output at any angle and distance is completed.

\section{Modeling experimental methods}

Experimental method is a very promising research method, which take advantage of different experimental test data, simplifying the complexity of the human body model, to shorten the study period. Experimental methods of human motion simulation requires a lot of experimental data, the use of sports biomechanics testing methods can be kinematic, kinetic and EMG information data. To obtain these data must be video, three-dimensional force plates, EMG and other equipment, but to get the full body motion test data, utilization of these devices must be synchronized, so the development of the experimental method is largely dependent on human movement tests and improve sensor technology [4].

Establish axes in the sweet spot, the ball was hit at the moment, it is assumed in the center of the sphere at the origin (as shown below), thereby obtaining $\mathrm{x}, \mathrm{y}, \mathrm{z}$ axis velocity components: $v_{x}, v_{y}, v_{z}$.

$$
\begin{aligned}
& \mathrm{v}_{x}=V \cdot \cos \theta_{x} \\
& \mathrm{v}_{y}=V \cdot \cos \theta_{y} \\
& \mathrm{v}_{z}=\sqrt{V^{2}-\mathrm{v}_{x}^{2}-\mathrm{v}_{y}^{2}}
\end{aligned}
$$

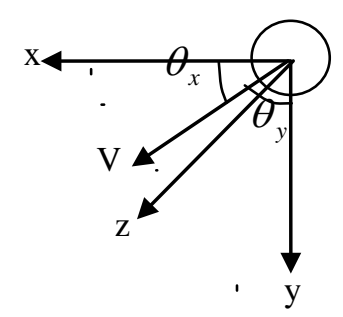

Ball success rate is the size of these three points, and the size of which is determined after the three velocity components in the player hit the ball, the ball has velocity (ie, the hitting power hitter and hitting direction) this problem requires by determining the angular range, so that the ball can fall into untenable area [4], thereby solving the problem can be attributed to the success of the ball fell to consider each case untenable region objective function,

$$
\begin{gathered}
\min \theta_{x}, \max \theta_{x}, \min \theta_{y}, \max \theta_{y} . \\
\left(L-l_{3}+l_{1}\right) \leq L_{x} \leq\left(L+l_{1}\right) \\
L_{y} \geq H
\end{gathered}
$$




$$
-l_{b} \leq L_{z} \leq\left(L-l_{b}\right)
$$

The ball fell to emulate the success of the rear untenable region.Mathematical model can be divided into two categories, one is the mechanism analysis, one is testing analysis. Mechanism analysis is based on the understanding of the real object properties, analyze the cause and effect relationship, and find out the rules to reflect the internal mechanism of the proposed model is often clear physical or practical significance [5]. Test analysis study as a "black box" system, the internal mechanism cannot seek direct output of the system can measure the input data as the basis for the use of statistical methods, in accordance with the guidelines determined in advance in a certain type of model selected a model best fits the data, this method is called system identification, also called black-box approach. Sometimes, the combination of both methods uses the mechanism of structural modeling to determine the parameters of the model using system identification [5] (shows in fig.1 ).

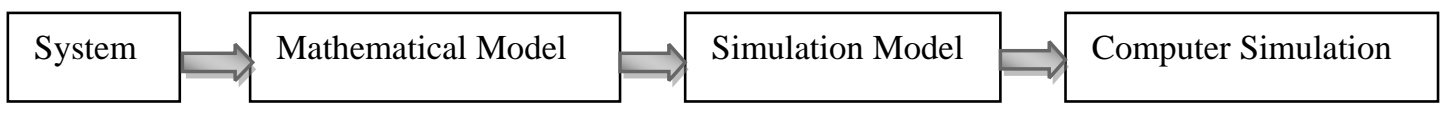

Fig.1 The simulation model implementation steps

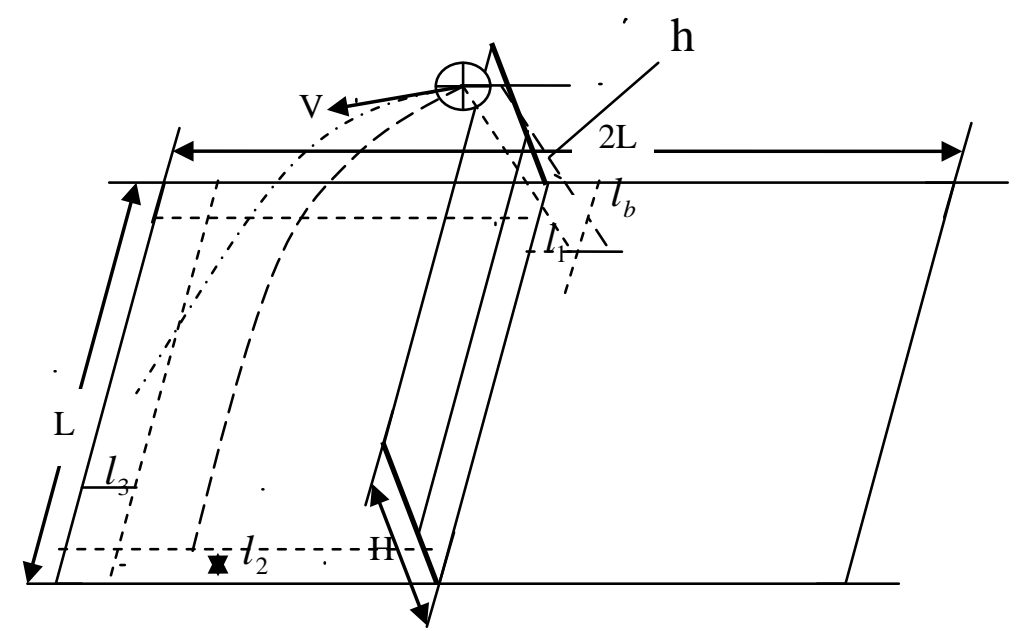

Fig.2 The ball fell to emulate the success of the rear untenable region

Ball-axis direction can be successfully fall back area, the ball values of the size range in the axial direction (in origin 0 to set the value of size), some scholars are trying to introduce some new research in the modeling process [6]. Ways and means shown in Fig 2.

From the chart analysis can be drawn: the range of $\theta_{x}, \theta_{y}$ variation must meet the following conditions.

$$
\begin{gathered}
\left(L-l_{3}+l_{1}\right) \leq L_{x} \leq\left(L+l_{1}\right) \\
L_{y} \geq H \\
-l_{b} \leq L_{z} \leq\left(L-l_{b}\right)
\end{gathered}
$$

The ball was hit to the ground from the process, by the vertical direction (axial) available:

$$
v_{x}=\frac{d_{y}}{d_{t}} \Rightarrow d_{y}=v_{y} \cdot d_{t}
$$

Compared with the traditional dynamics method, the above model in: (1) you do not need to understand the internal state of the simulation model, and will not produce errors due to the simplified model brought. (2) the body's movement control is a dynamic feedback process, the human body to complete an action must be dynamically balanced and coordinated control by visual, vestibular, feedback body, and the physical mechanics model can not simulate the dynamic control of the human body action [6]. 
Artificial neural networks as a powerful tool for modeling of nonlinear systems, not only simplifies the calculation of the amount of modeling, while also taking into account factors between systems [6], and EMG, kinematic and kinetic parameters of data can be the nonlinear relationship to model complex relationships between the neural network shown in Fig 3.

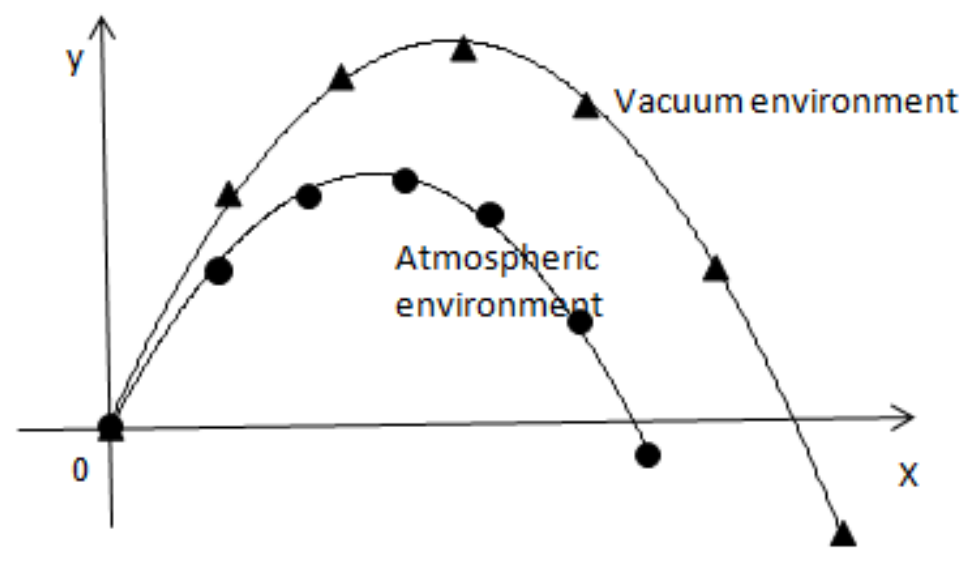

Fig.3Projectile Motion simulation results at atmospheric and vacuum

\section{Summary}

Virtual reality and simulation technology is an emerging science and technology and its applications in sport is just beginning, to be co-workers in the research and development of our sport, and now, virtual reality and simulation technology in the research, assisted instructional design, other aspects of simulation and analysis has shown great potential. It is used in modern volleyball techniques and tactics of teaching and training will have a broad impact on space and immeasurable.

\section{References}

[1] H.Y. Wang. On the Application of Physical Training Simulation of digital technology, Journal of Jilin Institute of Physical Education, 2007, pp. 11-14. (In Chinese)

[2] Q.Ch. Yu,Digital technology in sports teaching, Anhui Sports Science, 2005, pp.65-68. (In Chinese)

[3] J.D.Wang,Y.M. Liu,Digital technology applications in science and technology Olympics. North China University of Technology, 2005, pp.35-38. (In Chinese)

[4] X.J. Jiang,Application of digital technology Basketball in the, Sports and Health, 2009, pp.23-29. (In Chinese)

[5] J.L. Chen, Eduction digital technology in sport simulation application technology, sports science, 2009, pp.4-9. (In Chinese)

[6] Y. Huang, Talking about the digital technology, Science and Technology Information (academic studies), 2008, pp.34-39. (In Chinese) 